\section{From the authors:}

We thank P. Wijkstra and co-workers for their interest and comments regarding our paper, "Abdominal volume contribution to tidal volume as an early indicator of respiratory impairment in Duchenne muscular dystrophy" [1]. They wonder if our findings of progressive drop of abdominal contribution to tidal volume in Duchenne muscular dystrophy (DMD) are somewhat related to: 1) differences in forced vital capacity (FVC) between sitting and supine position; and 2) nocturnal hypercapnia. These questions are of great interest and they deal with three key aspects regarding the prognosis and the management of patients with DMD and other primary neuromuscular diseases, namely the noninvasive assessment of diaphragm function, the evaluation of sleep disordered breathing and the choice of the correct timing for the introduction of nocturnal noninvasive mechanical ventilation.

Unfortunately, we are not able to provide an answer based on supine FVC and/or carbon dioxide tension, because these measurements were not considered in our study. We have now calculated, however, for each control subject and DMD patient, the difference of the percentage abdominal contribution to tidal volume $(\Delta V \mathrm{ab} \%)$ between supine and seated position during spontaneous breathing at rest. As expected, the different diaphragm length and load in the two postures determined, in the healthy control subjects, a higher $\Delta V \mathrm{ab} \%$ in the supine position, with a difference between supine $\Delta V \mathrm{ab} \%$ and sitting $\Delta V \mathrm{ab} \%$, on average, equal to $28 \%$. In the four groups of DMD patients with increasing age, this difference was $25 \%, 20 \%, 16 \%$ and $-1.5 \%$. In the patients who showed nocturnal hypoxaemia it was, on average, equal to $-10 \%$.

These data reinforce the observation raised by P. Wijkstra and co-workers that differences between supine and seated position can provide useful indicators of diaphragmatic involvement in DMD. The point of our paper is that significant alterations are present even during quiet spontaneous breathing, and there is no need to ask the subject to perform a manoeuvre such as a FVC, which requires patient cooperation, is dependent on patient motivation and is frequently not tolerated by DMD patients in supine positions. Of course, the measurement methods used in our study are not available in all hospitals. However, our results suggest that technological developments should be addressed in order to provide simple but accurate measurement of abdominal kinematics during spontaneous breathing.

Another observation should be given in addition. At present, lung function evaluation in two different postures is not frequently used. Only few, partial and inconclusive studies are available and no clear indications on reference data are provided. RAGETTE et al. [2] suggested that a $>25 \%$ drop of inspiratory vital capacity upon shift from upright to supine position is an indication of diaphragm weakness. VARRATO et al. [3] measured seated and supine FVC in 38 patients with amyotrophic lateral sclerosis and found that seated-supine FVC difference is significantly greater in patients with complaints of dyspnoea, orthopnoea and daytime fatigue. FROMAGEOT et al. [4] studied a small group of patients with generalised neuromuscular disease and showed that the fall in vital capacity in the supine position was greater in the patients who had spontaneous paradoxical diaphragmatic motion and diaphragmatic weakness. Although these studies suggest that supine vital capacity may improve the poor sensitivity of spirometry to detect a moderate inspiratory muscle weakness, there is still a need for more definitive studies able to provide reference data eventually useful for patient assessment and clinical decision about noninvasive mechanical ventilation. The same applies to the observation that neuromuscular disease patients with nocturnal hypercapnia but daytime normocapnia may benefit from the introduction of nocturnal noninvasive ventilation before daytime hypercapnia develops [5]. Again, we need more definitive studies. The availability of objective measurements such as the one we describe should help us to design future studies addressed to better understand the mechanisms underlying the progressive involvement of respiratory muscles in neuromuscular disorders and the correct timing for the introduction of noninvasive mechanical ventilation.

\section{A. Aliverti*, A. Lo Mauro* and M.G. D'Angelo ${ }^{\#}$}

*Dipartimento di Bioingegneria, Politecnico di Milano, Milan, and "IRCCS E.Medea, Bosisio Parini, Italy.

Correspondence: A. Aliverti, TBM Lab, Dipartimento di Bioingegneria, Politecnico di Milano, P.zza L. da Vinci, 32, 20133 Milan, Italy. E-mail: andrea.aliverti@polimi.it

Statement of Interest: Statements of interest for A. Aliverti and A. Lo Mauro can be found at www.erj.ersjournals.com/site/ misc/statements.xhtml

\section{REFERENCES}

1 Lo Mauro A, D'Angelo MG, Romei M, et al. Abdominal volume contribution to tidal volume as an early indicator of respiratory impairment in Duchenne muscular dystrophy. Eur Respir J 2010; 35: 1118-1125.

2 Ragette R, Mellies U, Schwake C, et al. Patterns and predictors of sleep disordered breathing in primary myopathies. Thorax 2002; 57: 724-728.

3 Varrato J, Siderowf A, Damiano P, et al. Postural change of forced vital capacity predicts some respiratory symptoms in ALS Neurology 2001; 57: 357-359.

4 Fromageot C, Lofaso F, Annane D, et al. Supine fall in lung volumes in the assessment of diaphragmatic weakness in neuromuscular disorders. Arch Phys Med Rehabil 2001; 82: 123-128.

5 Ward S, Chatwin M, Heather S, et al. Randomised controlled trial of non-invasive ventilation (NIV) for nocturnal hypoventilation in neuromuscular and chest wall disease patients with daytime normocapnia. Thorax 2005; 60: 1019-1024.

DOI: $10.1183 / 09031936.00127310$ 